\title{
Conduites addictives en milieu hospitalier : particularités d'un service d'anesthésie
}

\author{
Is there a specific addiction to drug in the \\ anaesthetist population?
}

Pascal KINTZ*, Marion VILLAIN, Vincent CIRIMELE, Bertrand LUDES

Institut de Médecine Légale, 11, rue Humann - 67000 STRASBOURG

* Auteur à qui adresser la correspondance : Pascal KINTZ, Institut de Médecine Légale, 11, rue Humann 67000 STRASBOURG - Tél : 0390243349 - e-mail : pascal.kintz@wanadoo.fr

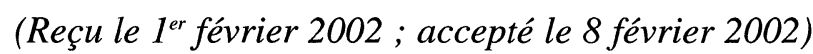

\section{RÉSUME}

Parmi les risques professionnels encourus par le personnel (médecins, infirmières) des services d'anesthésie, on cite fréquemment la transmission virale ou les allergies dues au latex ou à l'halothane. Plus sournois, plus discret et tellement moins médiatique, le risque de détournement de substances actives est une donnée difficilement quantifiable mais dont les conséquences dépassent largement le cadre hospitalier, comme en témoigne le retentissement judiciaire croissant. Les produits les plus souvent utilisés sont la morphine, le propofol, la kétamine, le thiopental, les dérivés du fentanyl et le midazolam, soit l'arsenal thérapeutique standard de ces services, à l'exclusion des curarisants, jugés trop dangereux. Les signes faisant évoquer une dépendance chimique sont les suivants : sujet prenant les gardes des autres, en particulier le week end lorsque la surveillance se relâche,

\section{SUMMARY}

Chemical dependency is a disease that can affect all professions. Among the health care professionals, anesthesiologists represent a specific group. Numerous factors have been proposed to explain the high incidence of drug abuse among anesthesiologists. These include : easy access to potent drugs, particularly narcotics, highly addictive potential of agents which they are in contact, and easy diversion of these agents since only small doses will initially provide an effect desired by the abuser. Opioids are the drugs of choice for anesthesiologists, and among them fentanyl and sufentanil are the most commonly used. Alcohol, mostly in older anesthesiologists, propofol, ketamine, thiopental and midazolam are also abused. In fact, all but quaternary ammonium drugs can be observed. Symptoms of addiction in the hospital include : unusual changes in behavior, desire to work alone, 
sujet ne prenant pas son repas de midi, sujet toujours volontaire pour finir les interventions des autres, sujet se rendant plus souvent aux toilettes que le reste du personnel, sujet travaillant ou souhaitant travailler pour des services très consommateurs en morphiniques comme la chirurgie thoracique, l'ensemble étant associé à une modification du comportement (isolement, somnolence, diminution des échanges verbaux ...). Si la recherche d'une solution en interne est souvent proposée, d'autres cas, en particulier lors de vols à répétition, ont des conséquences judiciaires. L'analyse toxicologique est alors confrontée à 2 types de problèmes, les propriétés pharmacologiques de ces produits et les difficultés analytiques pour les caractériser. En effet, il s'agit souvent de produits à $1 / 2$ vie courte, voire ultra-courte et dont les concentrations circulantes sont extrêmement faibles. Dans ces conditions, le laboratoire doit disposer des techniques séparatives les plus sensibles, comme la CG/SM/SM ou la CLHP/SM. Fréquemment les analyses sanguines et/ou urinaires sont prises en défaut. L'analyse des cheveux montre alors son intérêt, en augmentant la fenêtre de détection de quelques jours à plusieurs mois, selon la longueur des cheveux analysés (chaque cm représente la pousse d'environ 1 mois), tout en étant le reflet d'un abus à long terme. Par quelques exemples (infirmière décédée d'un mélange propofol-midazolam, médecins anesthésistes abusant de fentanyl, sufentanil, alfentanil, codeine, ou encore de midazolam) les auteurs feront le point sur les risques de toxicomanie chez le personnel hospitalier, les pratiques en terme de produits et enfin sur les approches analytiques, associant l'analyse des cheveux aux couplages chromatographie/spectrométrie de masse.

\section{MOTS-CLÉS}

Addiction, anesthésie, fentanyl, cheveux.

\section{Introduction}

Parmi les risques professionnels encourus par le personnel (médecins, infirmières) des services d'anesthésie, on cite fréquemment la transmission virale ou les allergies dues au latex ou à l'halothane. Plus sournois, plus discret et tellement moins médiatique, le risque de détournement de substances actives est une donnée difficilement quantifiable mais dont les conséquences dépassent largement le cadre hospitalier, comme en témoigne le retentissement judiciaire croissant. Cet article se propose de faire le point sur les connaissances de cette forme particulière d'addiction, en se focalisant essentiellement sur une approche médico-légale applicable par l'expert en toxicologie. Ainsi, seront abordés les thèmes suivants : épidémiologie, produits utilisés, profils de consommation, implications médico-légales, arsenal analytique et place des cheveux dans la mise en évidence d'une conduite addictive. De ce fait, les causes de cette toxicomanie, sa prévention, sa prise en charge et les éventuels traitements ne seront pas évoqués. Quatre expertises judiciaires serviront de support à notre démonstration. refuse of lunch relief or breaks, volunteer for extra cases, call, come in early and leave late, frequent restroom breaks, weight loss and pale skin, malpractice, behind on charts ... Toxicological investigations are difficult, as the drugs of interest are difficult to test for. In most cases, half-lifes of the compouds are short, and the circulating concentrations weak. It is therefore necessary to develop GC/MS/MS or LC/MS procedures to satisfy the criteria of identification and quantitation. In most cases, blood and/or urine analyses cannot document impairment. Hair analysis appears therefore as the unique choice to evidence chronic exposure. Depending the length of the hair shaft, it is possible to establish an historical record, associated to the pattern of drug use, considering a growth rate of about $1 \mathrm{~cm} /$ month.

Several real cases (nurse deceased from a propofol/midazolam mixture, anesthesiologists abusing fentanyl, sufentanil, alfentanil, codeine or midazolam) will document forensic investigations. The authors will then focus on the special place of hair analysis, associated to the more hyphenated analytical techniques.

\author{
KEY-WORDS \\ Addiction, anesthesiologist, fentanyl, hair.
}

\section{Données épidémiologiques}

Les données de la littérature sont peu nombreuses, l'essentiel étant rédigé par des auteurs américains. Nous n'avons trouvé qu'une seule revue générale écrite par un groupe français, de l'hôpital Tenon à Paris (1). Les données françaises sont donc quasi-inexistantes et cette absence illustre la difficulté à appréhender le problème. En 1973, l'association des médecins américains publiait un rapport (2) où 2,3 à $3,2 \%$ des médecins étaient considérés comme dépendants à l'alcool et 0,9 à 2,0\% toxicomanes à des substances illicites. Jusqu'en 1986, ces chiffres ont été en augmentation, avec 3,3\% des médecins et $5,2 \%$ des internes en médecine considérés comme toxicomanes (3). De nos jours, ces chiffres sont plutôt en régression, et en 1993, la prévalence de la toxicomanie a été évaluée à 1,3\% en Australie et en Nouvelle-Zélande chez les anesthésistes (4).

Les comparaisons entre les différentes spécialités médicales montrent un surcroît de risque de développer une addiction chez le personnel des services d'anesthésie (5). Par ailleurs, une sur-représentation des anesthésistes par rapport aux autres spécialistes de médecine est 
observée dans les programmes de désintoxication (1). Enfin, sur 2715 plaintes pour erreur médicale, déposées à l'encontre des anesthésistes américains entre 1977 et 1987, cinq dossiers étaient liés à une toxicomanie, dont 4 cas d'éthylisme chronique (6).

Ces données apparaissent superposables à celles, plus éparses, concernant les infirmières $(7,8)$.

\section{Produits utilisés}

Même s'il est très difficile d'établir une pratique générale, il semble que la toxicomanie en service d'anesthésie commence par un produit et qu'une dérive s'installe en quelques mois vers la polytoxicomanie (1).

Parmi les produits utilisés, l'éthanol correspond essentiellement à un abus des praticiens plus agés.

On retrouve surtout les narcotiques (fentanyl, sufentanil et à un degré moindre morphine), les benzodiazépines (surtout à visée hypnotique, comme le midazolam) et enfin certains produits moins courants comme le propofol (pourtant présenté comme sans pouvoir addictif), la kétamine (dont l'usage est compliqué par ses propriétés hallucinogènes, (9)) ou le sevoflurane. La péthidine, le dextromoramide ou le thiopental sont nettement moins utilisés de nos jours. A notre connaissance, il ne semble pas qu'il y ait eu détournement d'usage de GHB ou de buprénorphine dans le cadre d'une pharmaco-dépendance en milieu hospitalier.

Les curares, du fait de leurs propriétés pharmacologiques, ne sont jamais employés.

L'achat de ces produits au marché noir est exceptionnel (10), l'approvisionnement étant la plupart du temps assuré par un détournement hospitalier.

La mise à disposition facile d'agent ayant un fort potentiel addictif, comme le fentanyl ou le sufentanil apparaît comme un facteur de provocation, d'entretien ou de rechute de la toxicomanie (11). En outre, les doses actives étant faibles, la quantité à s'administrer, tout au moins au début, est réduite. Néanmoins, s'agissant de narcotiques propices à engendrer une tolérance rapide, l'intéressé devra fréquemment augmenter ses doses.

Toutes les voies d'administration sont possibles, comme les voies IV les plus inhabituelles, dans des veines cachées (en particulier pour dissimuler les points d'injection), la voie orale ou nasale pour les benzodiazépines ou la kétamine, ou encore les voies rectales ou sublinguales. Le côté tabou de l'injection parentérale ne semble pas exister.

\section{Profil de consommation}

La toxicomanie aux substances illicites apparaît comme plus fréquente chez les médecins anesthésistes que dans la population générale (11). Une des raisons invoquées est la plus grande disponibilité des narcotiques pour un usage quotidien. La course au produit, en particulier pour éviter le syndrome de manque n'est pas une caractéristique classique en milieu hospitalier. Le stress $(12,13)$, les pressions et les risques médicolégaux, la surcharge de travail, associés à l'exercice de l'anesthésie-réanimation qui peut être considérée comme une activité "toxicomanogène" (11) sont des facteurs favorisants le développement d'une conduite addictive.

Les rares études épidémiologiques démontrent une forte prédominance masculine, dont la moitié a moins de 35 ans. Il apparaît que les anesthésistes ont une plus grande curiosité pharmacologique que les autres médecins et sont donc plus facilement approchés par le goût du risque.

Les signes cliniques d'une addiction s'observent d'abord dans la vie sociale, puis à la maison et enfin sur le lieu du travail $(1,11)$. Il est alors possible de noter une alternance de période de dépression, d'angoisse, d'irritabilité avec des phases d'euphorie.

Les symptômes habituels d'une toxicomanie en service d'anesthésie sont les suivants :

- changement de comportement inhabituel au cours d'une même journée

- absences inexpliquées et répétées

- perte de poids et teint pâle

- sujet reprenant les gardes des autres, toujours d'accord pour faire des gardes le soir ou le week-end (lorsqu'il y a moins de surveillance)

- préférence pour la pratique de l'anesthésie en solitaire

- fréquentation assidue des toilettes par rapport aux autres collègues

- sujet sautant des repas, des pauses

- sujet d'accord pour finir les interventions des autres

- sujet travaillant dans des services très consommateurs d'antalgiques majeurs (comme la chirurgie thoracique ou cardiaque)

- sujet arrivant tôt le matin et partant tard le soir - plus rarement signes classiques d'un syndrome de manque

Les narcotiques peuvent être obtenus par substitution des produits prescrits, retransmission erronée sur l'ordonnancier ou le compte-rendu opératoire, utilisation des restes d'ampoules, sur-prescription et enfin échange du contenu des ampoules, en particulier par du sérum physiologique.

Des douleurs postopératoires excessives chez les patients 
pris en charge par un anesthésiste précis sont très évocatrices d'une anesthésie ou d'une analgésie incomplète et donc d'une administration partielle de la dose.

La période pendant laquelle une conduite addictive reste cachée peut être très variable : 1 à 6 mois pour le sufentanil, 6 à 12 mois pour le fentanyl, plus d'un an pour les autres substances injectables et enfin plus de 20 ans pour l'alcool éthylique (2).

\section{Implications médico-légales}

Une conduite addictive en milieu hospitalier fait essentiellement courir 3 types de risque, à l'individu toxicomane tant au niveau de sa santé personnelle que par des aspects réglementaires (sanction, radiation, instruction judiciaire pour détournement ou vol de stupéfiants ...), au patient et enfin à l'administration hospitalière.

Après la mise en évidence de leur toxicomanie, le risque de décès est élevé chez les médecins anesthésistes. Des chiffres de 10 à $16 \%$ sont rapportés (1). Il existe également une surmortalité par suicide ; le nombre de suicide chez les anesthésistes est 2 fois supérieur à celui des internistes (14). Le nombre de rechute, après retour en service hospitalier est impressionnant, il est donné à $19 \%$ chez les seniors (15) et à $66 \%$ chez les internes (16).

En règle générale, les risques pour les patients sont faibles. La qualité de l'anesthésie peut être mauvaise, engendrant des douleurs postopératoires importantes. Les accidents semblent être exceptionnels. Il est à noter que du fait de l'apparition retardée des signes cliniques d'une addiction sur le lieu du travail et donc d'une modification de comportement préjudiciable au patient, bien souvent une surveillance étroite a déjà été mise en place.

Enfin, au niveau administratif, l'hôpital peut voir sa responsabilité engagée du fait d'un manquement disciplinaire ou d'une erreur médicale. L'échelle des responsabilité est une cascade difficile à établir, mais il ne faut jamais oublier que la délivrance des stupéfiants suit des règles très strictes, sous la responsabilité d'un pharmacien, avec un ordonnancier tenu à jour. Les ratures dans ce livre doivent être expliquées, les ampoules et les doses doivent être comptabilisées et l'échange ampoule vide contre ampoule pleine est une réalité qui permet de prévenir au mieux toute dérive. En cas de casse d'une ampoule, le chef de service d'anesthésie est tenu de faire une déclaration par écrit auprès du pharmacien responsable.

\section{Aspects analytiques}

$\mathrm{Au}$ niveau sanguin, les concentrations circulantes des produits détournés sont généralement faibles, de l'ordre de 1 à $5 \mathrm{ng} / \mathrm{ml}$, parfois moins comme pour le sufentanil. D'autres produits sont volatils, comme le propofol ou le sevoflurane. Ils ont comme caractéristique pharmacologique une demi-vie généralement courte, ce qui complique d'autant l'analyse toxicologique. En France, il n'existe pas sur le marché de réactifs immuno-chimiques pour le dépistage des agents anesthésiques, à l'exclusion de la morphine et du midazolam, qui est très bien reconnu par les kits spécifiques des benzodiazépines. Dans ces conditions, l'analyste se doit de disposer d'un parc de matériel particulièrement impressionnant, comprenant la $\mathrm{CG} / \mathrm{SM}$ pour les opiacés, la $\mathrm{CG} / \mathrm{SM} / \mathrm{SM}$ pour les dérivés du fentanyl, la CG/SM avec introduction par espace de tête pour le propofol ou le sevoflurane et enfin la CG/SM avec ionisation chimique négative ou la CLHP/SM pour les benzodiazépines. Compte tenu des implications médico-légales évidentes et de l'importance de la responsabilité médicale, la spectrométrie de masse doit être la règle absolue dans tous les dossiers.

Dans la pratique, les résultats des analyses de sang ou d'urine sont décevants et ne permettent pas, dans de bonnes conditions, de mettre en évidence une éventuelle conduite addictive. Il n'est pas d'usage, du moins en France, d'effectuer de façon impromptue un prélèvement sur un collègue.

Le contrôle urinaire ne fait pas partie des propositions des associations de médecins pour favoriser le dépistage et une meilleure prise en charge (1).

Sur le plan médico-légal, seule l'analyse des cheveux permet d'apporter une réponse satisfaisante pour établir une éventuelle toxicomanie.

\section{Place des cheveux}

La décennie écoulée a confirmé l'intérêt majeur des cheveux comme marqueurs d'exposition chronique aux xénobiotiques. A présent, les applications de ces investigations débordent du champ purement judiciaire dans lequel elles avaient jusqu'alors été confinées, et s'imposent dans un nombre croissant de disciplines cliniques (17) ou dans le contrôle du dopage (18) .

Les poils sont des structures kératinisées propres aux mammifères, produites au niveau d'une invagination de l'épithélium épidermique, le follicule pilo-sébacé. Chacun de ces follicules représente une unité anatomique, constituée du poil proprement dit avec son bulbe pilaire, sa racine et sa tige, du follicule, d'une glande sébacée et d'un muscle horripilateur. L'homme adulte possède environ 5 millions de follicules pileux, dont un million, au niveau du scalp donnent naissance aux cheveux. La composition des poils est relativement 
variable : eau (4-13\%), protéines (85-93\%), lipides (1$3 \%)$ et minéraux $(0,2-0,8 \%)$.

Les poils se développent puis chutent de façon individuelle et cyclique, selon 3 phases : phase de croissance ou anagène (4 à 8 ans), phase de transition ou catagène ( 2 semaines) et phase de repos ou télogène ( 3 mois). A un instant donné, environ $85 \%$ des cheveux sont en phase anagène. On considère généralement que les cheveux au niveau du vertex poussent de 0,8 à 1,3 $\mathrm{cm} /$ mois, soit globalement $1 \mathrm{~cm} /$ mois (19).

Le mécanisme généralement proposé pour l'incorporation des xénobiotiques dans les cheveux consiste en une diffusion interne des substances du sang vers les cellules en croissance des bulbes pileux et une diffusion externe à partir des secrétions sudorales ou sébacées, selon la Figure 1.

En fusionnant pour former le cheveu, les cellules en croissance piègeraient les substances dans la structure kératinisée. Les cinétiques d'incorporation sont dépendantes des liaisons du xénobiotique incorporé à la mélanine, un pigment des cheveux. Il semble qu'il existe une différence quantitative d'incorporation suivant la couleur des cheveux, c'est à dire en fonction du degré d'oxydation de la mélanine. Par exemple, les cheveux foncés, présentant un degré d'oxydation plus important de la mélanine, concentrent ou retiennent plus fortement la cocaïne que les cheveux clairs, à doses administrées équivalentes (20).

Les substances mères sont présentes dans les cheveux où les poils ont des concentrations plus élevées que celles de leurs métabolites.

La fixation des xénobiotiques dans les cheveux pourrait également s'effectuer par le biais de l'environnement atmosphérique et concerne plus particulièrement les substances à l'état de particules en suspension. Ainsi, les substances fumées, comme le cannabis, le crack, ou même l'héroïne et la morphine peuvent se déposer sur toute la longueur du cheveu, ce qui nécessite une étape de décontamination des échantillons (lavages par un solvant organique). L'incorporation se faisant dans tous les poils, si les cheveux ne peuvent être prélevés ou sont manquants, d'autres poils conviennent également, comme les poils pubiens ou axillaires .

La stabilité des xénobiotiques une fois incorporés dans les cheveux semble tout-à-fait exceptionnelle. Il a ainsi été possible d'identifier de la cocaine dans les cheveux de momies péruviennes, vieilles de plusieurs centaines d'années.

Les cheveux sont généralement prélevés en vertex postérieur. Une mèche de 60 cheveux (diamètre d'un crayon) est largement suffisante. Celle-ci doit être prélevée le plus près de la peau, coupée au ciseau (ne pas arracher) et orientée racine-extrémité au moyen d'une cordelette, nouée $1 \mathrm{~cm}$ au dessus du niveau de la racine. La conservation est aisée; elle s'effectue en tube sec ou dans une enveloppe, à température ambiante.

Aujourd'hui, l'analyse segmentaire est un outil indispensable pour la justice et le corps médical afin de suivre l'évolution d'une toxicomanie ou la substitution par d'autres produits (21).

Néanmoins, les résultats quantitatifs, quels qu'ils soient, doivent être interprétés avec beaucoup de rigueur et de précautions, car si l'analyse segmentaire présente des avantages par rapport aux analyses traditionnelles dans le sang ou les urines (calendrier rétrospectif, fenêtre de détection, évolution de la consommation ...), il faut garder en mémoire que la croissance des

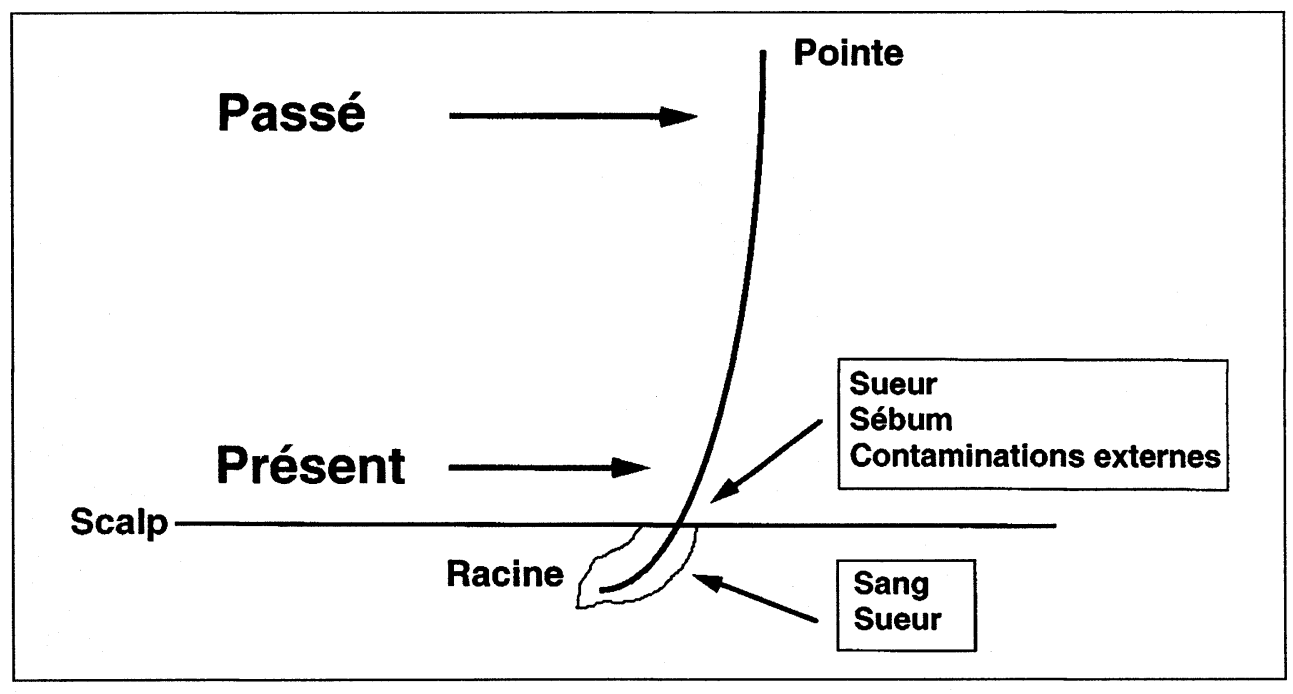

Figure 1 : Schéma simplifié d'un cheveu, avec les différents modes d'incorporation des xénobiotiques. 
cheveux n'est pas continue et que des phénomènes de migration à l'intérieur du cheveu peuvent affecter les concentrations.

\section{Applications judiciaires} $\operatorname{Cas} n^{\circ} 1$ (22)

Un médecin anesthésiste d'une cinquantaine d'années est soupçonné de consommer du fentanyl. Convoqué à plusieurs reprises par son administration pour effectuer des analyses urinaires, celles-ci ont toujours été négatives. Dans ces conditions, le médecin légiste a prélevé une mèche de cheveux de $3 \mathrm{~cm}$ et nous les a fait parvenir. Par CG/SM/SM, il a été possible d'identifier un usage chronique très important de fentanyl, puisque dosé à $644 \mathrm{pg} / \mathrm{mg}$. Une mise à la retraite anticipée a été proposée au médecin.

\section{$\operatorname{Cas~n}^{\circ} 2$}

Médecin exerçant dans un centre hospitalier général, le Dr G. est fortement suspecté de vol à répétition et de détournement de produits stupéfiants (ce qui sera vérifié lors d'une comparaison entre les compte-rendus opératoires et l'ordonnancier de la pharmacie). Dans le cadre d'une instruction pour abus de confiance et usage illicite de produits stupéfiants, le magistrat nous a fait parvenir une mèche de $6 \mathrm{~cm}$ de cheveux du médecin mis en cause. Selon la même procédure que précédemment, il a été possible d'identifier simultanément du fentanyl $(101 \mathrm{pg} / \mathrm{mg})$ et du sufentanil ( $2 \mathrm{pg} / \mathrm{mg})$. Ces résultats n'ont pas été contestés et l'instruction est en cours.

\section{Cas n ${ }^{\circ}$}

Dans le cadre d'une instruction contre un médecin de 40 ans, exerçant dans une clinique privée et mis en examen du chef d'usage de stupéfiants et de substances vénéneuses et de mise en danger d'autrui, nous recevons simultanément un échantillon urinaire et une mèche de cheveux. Toutes les analyses urinaires (effectuées par immuno-chimie et divers couplages chromatographiques avec la spectrométrie de masse) sont négatives, mais l'analyse des cheveux met en évidence de la codéine $(0,21 \mathrm{ng} / \mathrm{mg})$, de l'alfentanil $(30 \mathrm{pg} / \mathrm{mg})$ et du midazolam $(160 \mathrm{pg} / \mathrm{mg})$. L'interrogatoire permet de savoir que le début de la toxicomanie est liée à un état dépressif et que les produits étaient absorbés surtout le soir, injectés par voie intraveineuse. La radiation a été proposée.

\section{Cas n ${ }^{\circ} 4(23)$}

Une infirmière de 44 ans est retrouvée morte chez elle. Elle travaillait dans un service d'anesthésie. Sur place, les enquêteurs ont retrouvé un flacon vide d'Hypnovel (midazolam, $5 \mathrm{mg} / 5 \mathrm{ml}$ ). A l'exclusion de nombreux points d'injection et d'une congestion pulmonaire, l'autopsie est banale. L'analyse sanguine post-mortem met en évidence par CG/SM/ICN du midazolam $(45 \mathrm{ng} / \mathrm{ml})$ et par chromatographie gazeuse couplée à la spectrométrie de masse avec introduction par espace de tête du propofol $(39 \mathrm{ng} / \mathrm{ml})$. Ces 2 produits sont retrouvés par les mêmes techniques dans les cheveux, dont le premier segment ( 0 à $2 \mathrm{~cm}$ depuis la racine) donne les résultats suivants : $0,76 \mathrm{ng} / \mathrm{mg}$ (midazolam), $0,04 \mathrm{ng} / \mathrm{mg}(1-\mathrm{OH}-$ midazolam) et $1,39 \mathrm{ng} / \mathrm{mg}$ (propofol) et démontre donc une conduite addictive à long terme.

\section{Conclusion}

Toute baisse de la vigilance ou toute altération du jugement de l'anesthésiste met en péril la sécurité des patients. La toxicomanie en milieu anesthésique ne semble pas faire l'objet de travaux spécifique en France, et seules les expertises judiciaires permettent parfois d'appréhender le phénomène. L'analyste est souvent démuni pour caractériser les agents pharmacologiques responsables des addictions, du fait de la nécessité de disposer d'un arsenal analytique lourd. L'expérience des auteurs dans ce domaine a permis de conforter la place des cheveux dans la caractérisation formelle d'un usage chronique. Si le fentanyl et le sufentanil semblent être les 2 produits les plus couramment utilisés, le midazolam, la morphine et le propofol sont également souvent retrouvés. 


\section{Références}

1. Czernichow S., Bonnet F. Le risque de toxicomanie chez les médecins anesthésistes. Ann. Fr. Anesth. Réanim. $2000 ; 19: 668-74$.

2. American Medical Association Council on Mental Health. The sick physician : impairment by psychiatric disorders including alcoholism and drug dependency. JAMA $1973 ; 223$ : 684-7.

3. Mc Auliffe W.E., Rohman M., Santangelo S. et al. Psychoactive drug use among practicing physicians and medical students. N. Engl. J. Med. 1986 ; 315 : 805-10.

4. Weeks A.M., Buckland M.R., Morgan E.B., Myles P.S. Chemical dependence in anaesthetic registrars in Australia and New Zealand. Anaesth. Intensive Care $1993 ; 21: 151-5$.

5. Lutsky I., Hopwood M., Abram S.E., Cerletty J.M., Hoffman R.G., Kampine J.P. Use of psychoactive substances in three medical specialities : anaesthesia, medicine and surgery. Can. J. Anaesth. 1994 ; 41 : 561-7.

6. Sivarajan M., Posner K.L., Caplan R.A., Gild W.M., Cheney F.W. Substance abuse among anesthesiologists. Anesthesiology $1994 ; 80: 704$.

7. Bell D.M., McDonough J.P., Ellison J.S., Fitzhugh E.C. Controlled drug misuse by certified registered nurse anesthetists. AANA J. 1999 ; 67 : 133-40.

8. Griffith J. Substance abuse disorders in nurses. Nurs. Forum $1999 ; 34: 19-28$.

9. Moore N.N., Bostwick J.M. Ketamine dependence in anesthesia providers. Psychosomatics 1999 ; 40 : 356-9.

10. Brooke D., Edwards G., Taylor C. Addiction as an occupational hazard : 144 doctors with drug and alcohol problems. Br. J. Addict. 1991 ; 86 : 1011-6.

11. Chandon M. Toxicomanie et addictions en milieu anesthésique : sortir du non-dit. Ann. Fr. Anesth. Réanim. $2000 ; 19: 640-2$.

12. Jackson S. The role of stress in anaesthetists' health and well-being. Acta Anaesthesiol. Scand. 1999 ; 43 : 583602.
13. Kam P.C. Occupational stress in anaesthesia. Anaesth. Intensive Care 1997 ; 25 : 686-90.

14. Alexander B.H., Checkoway H., Nagahama S.I., Domino K.B. Cause-specific mortality risks of anesthesiologists. Anesthesiol. 2000 ; 93 : 922-30.

15. Arnold W.P. Substance abuse survey in anesthesiology training programs : a brief summary. ASA Newsl. 1995; $59: 12-3$.

16. Talbott G.D., Gallegos K.V., Wilson P.O., Porter T.L. The medical association of Georgia's impaired physician program. Review of the first 1000 physicians : analysis of speciality. JAMA $1987 ; 257: 2927-30$.

17. Goullé J.P., Kintz P. Un nouveau moyen d'investigation biologique : l'analyse des cheveux. Intéret en pratique médicale. Rev. Med. Interne 1996 ; 17 : 826-35.

18. Kintz P. Quelle place pour les cheveux dans la lutte contre le dopage ? Ann. Toxicol. Anal. $2000 ; 12$ : 49-55.

19. Tracqui A. Le poil : structure et physiologie. Rev. Fr. Lab. $1996 ; 282: 19-23$

20.Henderson G.L., Harkey M.R., Zhou C., Jones R.T., Jacob P. Incorporation of isotopically labeled cocaine and metabolites into human hair : 1 . Dose-response relationships. J. Anal. Toxicol. $1996 ; 20: 1-12$.

21. Pragst F., Rothe M., Spiegel K., Sporkert F. Illegal and therapeutic drug concentrations in hair segments - a timetable of drug exposure ? Forensic Sci. Rev. 1998 ; 10 : 81111.

22. Villain M., Cirimele V., Ludes B., Kintz P. Toxicomanie d'un anesthésiste au fentanyl : la preuve formelle par analyse des cheveux en utilisant la CPG/SM/SM. Ann Toxicol. Anal. $2001 ; 13$ : 49-53.

23. Cirimele V., Kintz P., Doray S., Ludes B. Determination of chronic abuse of the anaesthetic agents midazolam and propofol as demonstrated by hair analysis. Int. J. Leg. Med. $2002 ; 116$ : 54-57. 